\title{
Atomic-scale tip-sample interactions and contact phenomena
}

\author{
S. Ciraci \\ Department of Physics, Bilkent University, Bilkent 06533 Ankara, Turkey \\ and \\ Zurich Research Laboratory, IBM Research Division, 8803 Rüschlikon, Switzerland
}

Received 11 August 1991

\begin{abstract}
Tip-sample interactions become crucial owing to increased overlap at small tip-sample separation. The potential barrier collapses before the point of maximum attraction on the apex of the tip, but the effective barrier may remain significant owing to the strong confinement of current-carrying states to the constriction between tip and sample. At such separations the perpendicular tip force is still attractive and determined by ion-ion repulsion and redistribution of electronic charge. Electronic states are modified by the tip-induced perturbation of the potential in the vicinity of the tip. Self-consistent calculations reveal that local properties, such as elastic deformation, effective height and width of the tunneling barrier, electronic states and attractive tip force are site-dependent and reversible on the atomic scale. Numerical results suggest a relation between the perpendicular tip force and barrier height as a function of separation. A mechanical contact is formed with relatively strong bonds at separation near the point of zero force gradient. Whether the effective potential can collapse and hence the first channel can open to allow a transition from tunneling to ballistic conduction, and whether the conductance can show quantized steplike changes with increasing plastic deformation depends on material properties.
\end{abstract}

\section{Introduction}

During the past decade, scanning tunneling microscopy (STM) [1] has developed into a powerful experimental technique to probe local electronic structure without invoking periodicity. In the conventional view of STM the tip-sample separation $z$ is assumed sufficiently large to allow only weak coupling between their electronic states. In this case, the tunneling can be described by the transfer Hamiltonian approach in terms of the one-electron states of the bare electrodes (tip and sample), and the tunneling current is exponentially dependent on the separation. Accordingly, STM probes the weak overlap between the wave functions of tip and sample, and can yield resolution down to the atomic scale. The electronegativity difference between the tip and sample leads to a transfer of charge and thus to an electrostatic interaction. In addition, depending on the shape of the tip, strong Van der Waals (VdW) forces may act but are expected to be essentially uncorrugated on the atomic scale. Depending on the bias polarity, tunneling occurs either from filled or towards empty states of the sample and can reveal the spectrum of the sample surface in the range of energy $E_{\mathrm{F}}-e V \leq \epsilon \leq$ $E_{\mathrm{F}}+e V\left(E_{\mathrm{F}}\right.$ and $V$ being Fermi energy and bias voltage, respectively). New techniques, such as ballistic electron emission microscopy [2] (to probe interfaces and to provide spatial and energy resolution of the scattering process) and field emission of electrons from an atomically sharp tip [3] (to obtain a stable and well collimated e-beam), are also derived from STM. All these modes of STM operation are performed at large separations with nearly independent electrodes, and are identified as the conventional tunneling regime.

In some typical operating modes of STM [4], the observation of force variation of the order of $10^{-9} \mathrm{~N}$ (or $\sim 1 \mathrm{eV} / \AA$ ) has indicated significant tip-sample interactions $[5,6]$. In several other studies, the tip-sample separation was purposely set small to enhance the tip-sample interaction, 
and hence to modify the electronic and atomic structure of electrodes even irreversibly [7]. Indeed, atomic force microscopy (AFM) [8] probes the significantly strong forces exerted by the sample on a tip attached to a minute cantilever. In the mesoscopic system which becomes realized upon atomic contact between tip and sample the quantum-scale conductance $\left(\sim 2 e^{2} / h\right)$ was observed [9]. It is now expected that modifications of atomic and electronic structure with strongly interacting tip, and mesoscopic phenomena derived thereof, will dominate several studies using STM and AFM in the next decade. The present study is intended to be a concise theoretical analysis of tip-sample interactions at small separation with an emphasis on two new regimes of operation beyond that of conventional tunneling.

\section{Tip-sample interaction effects}

As the distance between a tip and sample is decreased the overlap of the wave functions of the electrodes increases and several interrelated atomic-scale interaction effects then come into play as suggested by investigations of the transi- tion from tunneling to electrical and mechanical contact [6,9-13]. The potential barrier between tip and sample is gradually lowered, which causes significant rearrangements of the charge density $[5,6]$. This, in turn, induces an attractive (bonding) interaction or adhesion energy leading to short-range attractive forces. Responding to the latter, the ions of the tip and sample are displaced even before plastic deformations set in. A few ångströms before mechanical contact, reversible local electronic and structural modifications are therefore expected. The potential barrier collapses [6] before the point of maximum attraction on the apex of the tip. This regime at intermediate separations is characterized by significant electronic interaction and occurs at separations smaller than that of conventional tunneling.

Upon further approach of the tip a mechanical contact is eventually formed through strong bonds with sample atoms. If the cross section of the contact is sufficiently large the effective barrier can also collapse, leading to a ballistic transport. Hence, the operation of STM in this range of very small separations reveals a different regime, in which the character of transport undergoes a

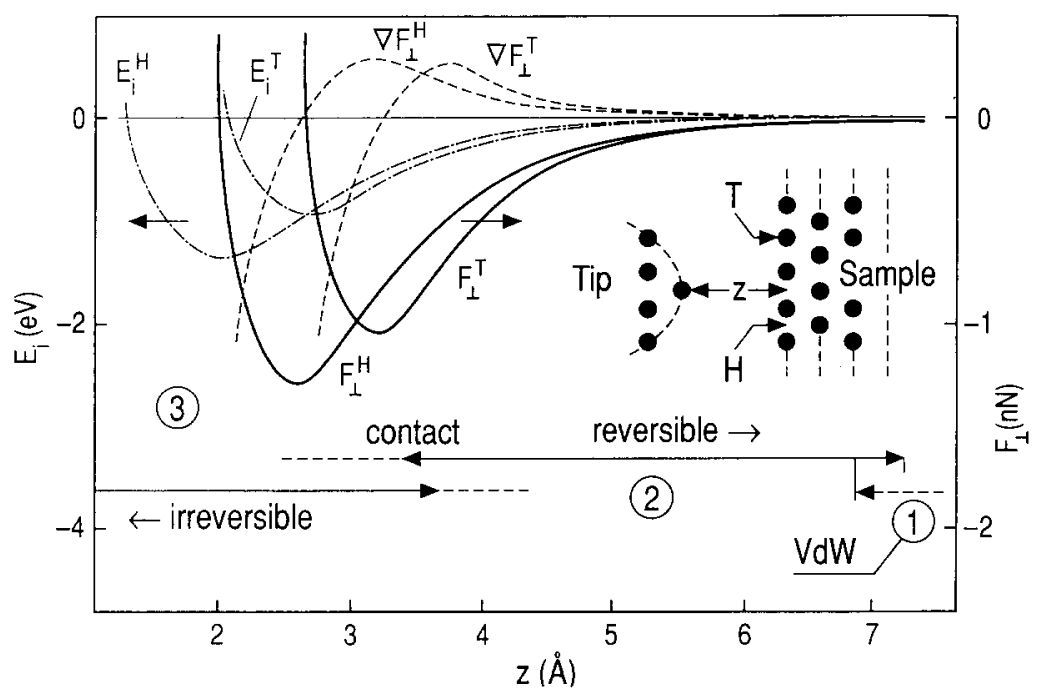

Fig. 1. Interaction energy $E_{\mathrm{i}}$, perpendicular force $F_{\perp}$, and force gradient $\nabla F_{\perp}$ (in arbitrary units) versus separation $z$ calculated for an Al(001) sample and tip at the hollow $(\mathrm{H})$ and top $(\mathrm{T})$ sites. Ranges of $z$ corresponding to three regimes of STM are indicated. 
qualitative change. Irreversible deformations are then also expected in the vicinity of the tip. We identify this regime as that of mechanical contact.

In fig. 1, these two regimes (i.e. mechanical and electrical contact regimes) beyond that of conventional tunneling are schematically represented with reference to physical quantities such as the interaction energy $E_{\mathrm{i}}$, the perpendicular component of the tip force $F_{\perp}$, and its gradient $\nabla F_{\perp}$. Of course, the extent of these regimes varies depending on the electronic and structural properties of the tip and sample. Besides, the transition between adjacent regions is not sharp. Nevertheless, mechanical contact occurs when $z$ is smaller than the separation where $\nabla F_{\perp}(z)$ has a maximum. The approach of a tip closer than the point of equilibrium $\left(z=z_{\mathrm{e}}\right)$ corresponding to the minimum of $E_{\mathrm{i}}$ results in a plastic (irreversible) mechanical deformation. The second regime, i.e. electronic contact, occurs roughly between mechanical contact and the conventional tunneling regime (i.e. between the strongly corrugated, short-range force region and the long-range $\mathrm{VdW}$ force region). Tip-induced mechanical and electronic deformations in this intermediate regime are reversible.

In our work the physical quantities, $E_{i}, F_{\perp}$ and $\nabla F_{\perp}$ in fig. 1 have been extracted from standard self-consistent field (SCF) pseudopotential calculations using the local density approach (LDA). It is known that LDA calculations are convenient for obtaining the SCF potential energy and charge density at small $z$, and for revealing interactions on the atomic scale but fail to describe long-range interactions. In our calculations either an $\mathrm{Al}(001)$ or an $\mathrm{Al}(111)$ slab is chosen as the sample, while the blunt (flat) and sharp tips are represented by an $\mathrm{Al}$ slab or an $\mathrm{Al}$ pyramid, respectively. Then, the properties of the entire system are computed in a supercell with periodic boundary conditions. The total energy and atomic forces are calculated in the momentum representation [14] for different lateral and perpendicular positions of the tip. The variation of the force gradient with separation $z$ is extracted from the derivative of $F_{\perp}(z)$, which is rather inaccurate but conceptually useful to display.
Owing to the limited size of the supercell and discretization in the wave-vector space, the LDA calculations described above are not convenient for calculating the current $I$ or conductance $\sigma$. However, $I$ or $\sigma$ corresponding to a fixed separation $z=z_{0}$ can be calculated for free electrons incident on a parameterized model potential between tip and sample fitted to the above-mentioned SCF calculations [13],

$$
\begin{aligned}
V\left(r, z_{0}\right)= & \phi_{\mathrm{m}}\left(z, z_{0}\right)+\alpha\left(z, z_{0}\right) \rho^{2} \\
& \times \Theta\left(z+d_{1} / 2\right) \Theta\left(z-z_{0}+d_{2} / 2\right) .
\end{aligned}
$$

Here $\rho=x^{2}+y^{2}, d_{1}$ and $d_{2}$ are half the interlayer distances along the $z$-axis at each electrode. The maximum of $\phi_{\mathrm{m}}\left(z, z_{0}\right)$ coincides with the saddle point of the three-dimensional potential, and the barrier height is $\Phi_{\mathrm{b}}=\max \left\{\phi_{\mathrm{m}}\left(z, z_{0}\right)\right\}-$ $E_{\mathrm{F}}$. The form of this model potential is in compliance with our SCF results and allows a separable solution of the Schrödinger equation. Having the parameterized form of the potential and treating tip and sample in the free-electron approximation, $\sigma$ is obtained by evaluating the expectation value of the current operator in terms of current-carrying states, and integrating it over the Fermi sphere. The current-carrying states, in turn, are calculated by a transfer matrix method [15]. This method has a wide range of applications covering tunneling as well as ballistic transport, even including the field emission of electrons.

Fig. 1 clearly shows that differences (corrugation) in $E_{\mathrm{i}}, F_{\perp}, \nabla F_{\perp}$ between the top site and hollow site positions of the tip are very small in the conventional tunneling regime, but gradually become pronounced as $z$ decreases. The minimum of $E_{\mathrm{i}}(z)$ at $z_{\mathrm{e}}$, where strong bonds are formed with sample atoms, varies depending on the tip position. Owing to additional attraction primarily due to $\mathrm{VdW}$ interaction with atoms behind the tip apex, this distance is expected to be smaller than that corresponding to a small model tip. Therefore, it is argued that the outermost tip atoms can feel strong repulsive interaction even while the $F_{\perp}$ is attractive [6]. In the 
mechanical contact regime, $E_{\mathrm{i}}(z)$ passes through a minimum and increases with decreasing $z$, while $F_{\perp}$ passes through zero and becomes increasingly repulsive even for the flat metal surface studied. A corrugation of $\sim 0.5 \AA$ is predicted for a tip under fixed load $F_{\perp}$ in the repulsive range. The component of $F_{\perp}$ acting on a given tip atom consists of nearly compensating ion-ion repulsion and ion-electron attraction. For $z<z_{\mathrm{e}}$ ion-ion repulsion exceeds the ion-electron attraction and thus yields a repulsive force. In the strong repulsive force region at very small $z$, ion-ion repulsion considerably exceeds the magnitude of ionelectron attraction at the top site and is also larger than ion-ion repulsion at the hollow site. Consequently, $F_{\perp}$ at the top site is larger than that at the hollow site as shown in fig. 1. In the conventional constant repulsive force mode of AFM the tip feels the ionic repulsion and therefore images atomic sites as maxima. However, as $z$ increases, the magnitude of ion-electron attraction decays more slowly than ion-ion repulsion, and $F_{\perp}$ changes sign and becomes attractive. This is caused primarily by the bonding charge density accumulated between tip and sample. In an intermediate range the difference between ion-ion repulsion at the top and the hollow site is still significant, and hence the attractive $F_{\perp}$ at the top site is smaller than that at the hollow site. For larger separations the electron-ion attraction dominates the ion-ion repulsion [6]. The resulting crossing of the force curves is material dependent and thus occurs at different $z$.

Our earlier calculation [5,6] illustrated how, upon approaching from the conventional tunneling regime, the overlap of the tip and sample wave functions $\left(\psi_{\mathrm{t}}\right.$ and $\left.\psi_{\mathrm{s}}\right)$ near $E_{\mathrm{F}}$ increases. As a result, the electronic structure of electrodes is perturbed depending on the value of the hopping matrix element, $\left\langle\psi_{\mathrm{t}}\left|\mathscr{H}_{\mathrm{t}+\mathrm{s}}\right| \psi_{\mathrm{s}}\right\rangle$, and the charge (corresponding to the bonding combination) between tip and sample is enhanced. If the barrier of the potential between electrodes is finite, the hopping matrix element is closely related to the tunneling matrix element [16]

$M=\frac{1}{2} \int\left(\psi_{\mathrm{t}} \nabla \psi_{\mathrm{s}}-\psi_{\mathrm{s}} \nabla \psi_{\mathrm{t}}\right) \cdot \mathrm{d} S$
This simple argument suggested a relation, such as $F_{\perp} \propto \kappa \exp (-\kappa z)$ [17]. Here the inverse decay length in atomic units is given by $\kappa=\sqrt{\Phi_{\mathrm{b}}}$. Recent extensive calculations confirm that such a relation between force and barrier height is indeed satisfied as long as $\Phi_{\mathrm{b}}$ is finite [18].

The anomalous corrugation observed on close-packed metal surfaces is a typical example for the reversible tip-sample interaction effects in the intermediate regime. The observed STM corrugation of the nominally flat (111) surfaces of $\mathrm{Al}$ was found to be much larger than one could infer from the charge density of the free surfaces [19]. Various models have been proposed to explain this anomalous effect. In the range where attraction increases with decreasing $z$ the tip-induced elastic deformation would reduce the corrugation of STM [6]. However, our SCF calculations for an $\mathrm{Al}$ tip and $\mathrm{Al}(111)$ sample showed that the corrugation of the surface potential is enhanced by the tip-induced modifications of the electronic structure [20]. As a result, the surface potential imaged by an atomically sharp tip leads to large corrugation which was found consistent with measurements [19] within computations based on eq. (1). This effect occurs over a certain range of $z$, but disappears at large separations.

In fig. 2, the contour plots of the SCF potential in the vicinity of an atomically sharp tip are shown for $z_{0}=4.8 \AA$. Already at this separation the potential barrier $\Phi_{\mathrm{b}}$ collapses and a classically allowed constriction with $V(r)<E_{\mathrm{F}}$ is formed between the outermost sample and tip atom at the top site. However, as seen from the schematic description of $V(r)$ in fig. $2 b$, the mean transverse momentum of current-carrying states is increased since they are confined to the narrow constriction at the saddle point. Consequently, the energy $\epsilon_{1}$ of the lowest eigenstate of $\mathscr{H}_{t+\mathrm{s}}$ in the constriction lies above $E_{\mathrm{F}}$ and acts as an effective barrier $\Phi_{\text {eff }}$ for $z$-variations of the wave functions. This situation may occur for an atomically sharp tip. Since the opening of the first propagation channel is delayed, tunneling persists even if $\Phi_{\mathrm{b}}<0$. Our simple estimates from the SCF potential of the $\mathrm{Al}$ tip and $\mathrm{Al}(111)$ sample indicate that even for $z=4 \AA$ the effective barrier is significant and site-dependent [6]. As men- 


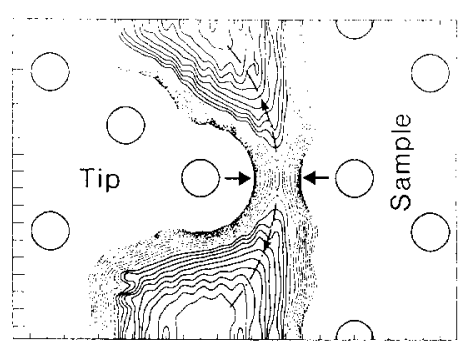

a

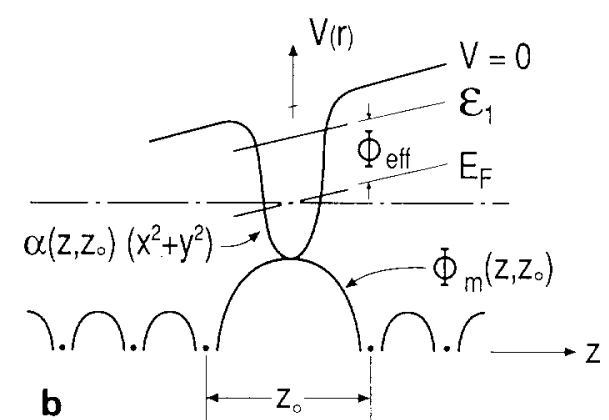

Fig. 2. (a) Counter plots of potential energy $V(r)$ calculated self-consistently for $\mathrm{Al}$ tip and $\mathrm{Al}(111)$ sample at the separation $z_{0}=5.3 \AA$. Solid and dotted curves correspond to $\left.V(r)\right\rangle$ $E_{\mathrm{F}}$ and $V(r)<E_{\mathrm{F}}$, respectively. The potential energy increases in the directions indicated by small arrows. (b) Schematic description of the above potential $V(\boldsymbol{r})$ with $z$-dependent and $x y$-dependent parts at the saddle point. $V=0$, $\epsilon_{1}, E_{\mathrm{F}}$, and $\Phi_{\text {eff }}$ indicate vacuum level, first subband energy, Fermi energy and effective barrier, respectively.

tioned above, $\epsilon_{1}$ drops and dips below the Fermi level (and hence the first conduction channel is opened) if the constriction becomes wide enough. This is only expected to happen at small $z$ following an irreversible plastic deformation.

Different regimes in the operation of STM have been discussed so far in terms of interaction energy and force between tip and sample. Next we discuss how these regimes are related to the conductance [9]. To this end, eq. (1) was fitted to the SCF potential between tip and sample, and the conductance $\sigma$ is calculated as a function of separation [13]. The results are presented in fig. 3 . For large $z$ we see that $\log _{10} \sigma(z)$ varies linearly with $z$. This behavior is characteristic of the tunneling conductance and occurs in the conventional tunneling as well as in the far end of the electronic contact regime, where $\kappa$ is still significant. As $z$ continues to decrease in the electronic contact regime one sees a deviation from the originally linear relationship. Finally, when $z$ decreases to $\sim z_{\mathrm{e}}$, the effective length of the constriction $d$ stops varying, although approach of the tip continues. In this situation, different behaviors for $\sigma$ may be encountered depending on various factors [13]. If the area of contact is small for an atomically sharp tip and $\Phi_{\text {eff }}$ is still finite, or if an oxide layer is located between tip and sample the conductance saturates over a small interval. Once $z \simeq z_{\mathrm{e}}$, plastic deformation takes place upon further approach of the tip; due to the increased contact area, $\Phi_{\text {eff }}$ collapses and ballistic transport sets in. Quantum size effects are expected to be crucial since the cross section of the constriction is comparable to the Fermi wavelength $\lambda_{\mathrm{F}}$ [11]. However, a quantization of $\sigma$ with well defined plateaus at multiples of $z e^{2} / h$ should not be resolved due to the shorter length

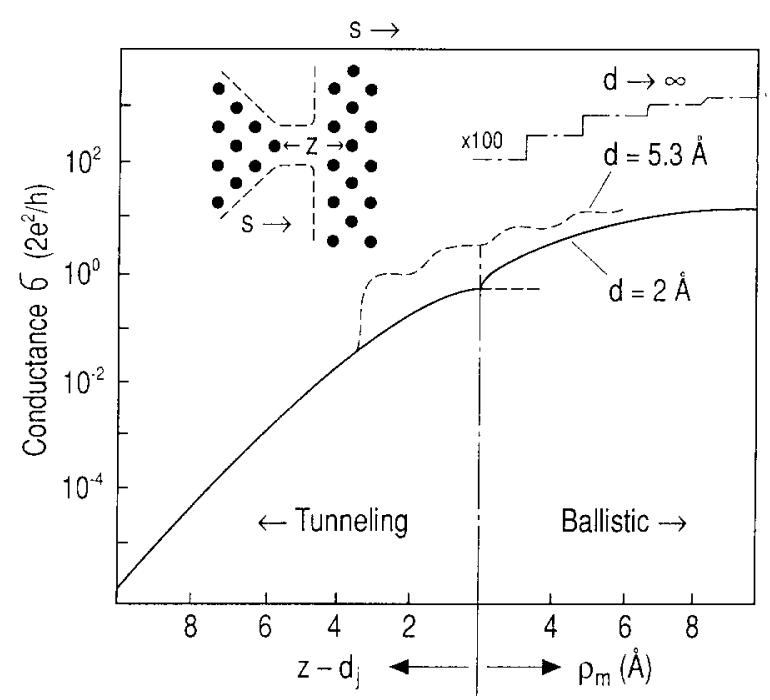

Fig. 3. Conductance, $\sigma$ versus separation $z$ or apparent displacement $s$ (in arbitrary units) of an $\mathrm{Ag}$ tip above an $\mathrm{Ag}$ sample showing also the transition from the tunneling to ballistic conduction. The term $\rho_{\mathrm{m}}$ is the radius of the constriction at $E_{\mathrm{F}}$. Quantum features are smeared out in the ballistic transport corresponding to $d=2 \AA$ shown by solid lines. Quantum features become apparent at the dashed line corresponding to $d=5.3 \AA\left(=\lambda_{\mathrm{F}}\right)$. Dash-dotted line shows sharp quantum steps in the constriction of infinite length. 
of the constriction ( $d=2 \AA$ ). However, if plastic deformation occurring leads to a longer effective length of the constriction $\left(d \simeq \lambda_{\mathrm{F}}\right)$ and if the width also increases uniformly due to the continuing displacement of the tip the quantum jumps should become less smeared out. This situation is, however, an unlikely possibility. Another more plausible explanation for the oscillations observed in $\sigma(z)$ beyond mechanical contact [9] is that the effective contact area changes abruptly, as it encompasses a set of atoms whose number increases stepwise as the tip is pushed into the sample.

\section{Conclusions}

Based on the results of SCF calculations of total energy, force, and potential energy for model tip-sample systems, three regimes are identified in the operation of STM and AFM. In the electronic contact regimes, perpendicular force and barrier height are site-dependent. The corrugation of the surface potential is enhanced by the tip-induced modifications of electronic structure. It is shown that the perpendicular force and barrier height are interrelated as long as the latter is positive. Beyond mechanical contact quantum features in the tunneling conductance versus tip displacement are smeared out due to the short length of the resulting constriction and, together with other properties, are strongly affected by irreversible plastic deformations effects.

\section{Acknowledgements}

I thank my collaborators, Drs. A. Baratoff, I.P. Batra and E. Tekman, for their valuable contributions to joint work on the tip-sample effects discussed in this paper. This work is supported by a Joint Study Agreement between the IBM Zurich Research Laboratory and Bilkent University.

\section{References}

[1] G. Binnig, H. Rohrer, Ch. Gerber and E. Weibel, Phys. Rev. Lett. 49 (1982) 57

[2] L.D. Bell, M.H. Hecht, W.J. Kaiser and L.C. Davis, Phys Rev. Lett. 64 (1990) 2679.

[3] H.W. Fink, W. Stocker and H. Schmid, Phys. Rev. Lett. $65(1990) 1204$.

[4] U. Dürig, O. Züger and D.W. Pohl, Phys. Rev. Lett. 65 (1990) 349;

F.O. Goodman and N. Garcia, Phys. Rev. B 43 (1991) 4728.

[5] E. Tekman and S. Ciraci, Phys. Rev. B 40 (1989) 10286.

[6] S. Ciraci, in: Basic Concepts and Applications of Scanning Tunneling Microscopy and Related Techniques, Vol. E184, Eds. H. Rohrer, N. Garcia and J. Behm (Kluwer. Dordrecht, 1990) p. 119;

S. Ciraci and I.P. Batra, Phys. Rev. B 36 (1987) 6194; I.P. Batra and S. Ciraci, J. Vac. Sci. Technol. A 6 (1988) 313 ;

S. Ciraci, A. Baratoff and I.P. Batra, Phys. Rev. B 41 (1990) 2763; Phys. Rev. B 42 (1990) 7618.

[7] J.M. Soler, A.M. Baro, N. Garcia and H. Rohrer, Phys. Rev. Lett. 57 (1986) 444;

see also F. Abraham, I.P. Batra and S. Ciraci, Phys. Rev. Lett. 60 (1988) 1314;

F. Abraham and I.P. Batra, Surf. Sci. 209 (1989) L125.

[8] G. Binnig, C.F. Quate and Ch. Gerber, Phys. Rev. Lett. $56(1986) 930$.

[9] J.K. Gimzewski and R. Möller, Phys. Rev, B 36 (1987) 1284.

[10] J.B. Pethica and W.C. Oliver, Phys. Scr. 19A (1987) 61.

[11] N. Garcia, unpublished.

[12] N.D. Lang, Phys. Rev. B 36 (1987) 8173; B 37 (1988) 10395.

[13] S. Ciraci and E. Tekman, Phys. Rev. B 40 (1989) 11969.

[14] I.P. Batra, S. Ciraci, G.P. Srivastava, J.S. Nelson and C.Y. Fong, Phys. Rev. B 34 (1986) 8246.

[15] E. Tekman and S. Ciraci, Phys. Rev. B 39 (1989) 8772; Phys. Rev. B 43 (1991) 7145

[16] F. Flores, A.M. Rodero, E.C. Goldberg and J.C. Duran, Nuovo Cimento 10 (1988) 303.

[17] C.J. Chen, J. Phys. (Cond. Matter) 3 (1991) 1227.

[18] S. Ciraci, E. Tekman, M. Gökcedag, I.P. Batra and A. Baratoff, Ultramicroscopy 42-44 (1992) 163.

[19] J. Winterlin, J. Wiechers, H. Brune, T. Gritsch, H. Höfer and R.J. Behm, Phys. Rev. Lett. 62 (1989) 59.

[20] E. Tekman and S. Ciraci, Phys. Rev. B 42 (1990) 1860; for a different explanation, see also: G. Doyen, E. Koetter, J.P. Vigneron and M. Scheffer, Appl. Phys. A 51 (1991) 281 